ITP-UU-03/45

SPIN-03/28

TUW-03-27

hep-th/0309220

\title{
Instanton Solutions for the Universal Hypermultiplet ${ }^{\dagger}$
}

\author{
Marijn Davidse ${ }^{1}$, Mathijs de Vroome ${ }^{1}$, Ulrich Theis ${ }^{2}$ and Stefan Vandoren ${ }^{1}$ \\ ${ }^{1}$ Institute for Theoretical Physics and Spinoza Institute \\ Utrecht University, 3508 TD Utrecht, The Netherlands \\ M.Davidse, M.T.deVroome, S.Vandoren@phys.uu.nl \\ ${ }^{2}$ Institute for Theoretical Physics, Vienna University of Technology, \\ Wiedner Hauptstrasse 8-10, A-1040 Vienna, Austria \\ theis@hep.itp.tuwien.ac.at
}

\begin{abstract}
We expand our previous analysis on fivebrane and membrane instanton solutions in the universal hypermultiplet, including near-extremal multi-centered solutions and mixed fivebrane-membrane charged instantons. The results are most conveniently described in terms of a double-tensor multiplet.
\end{abstract}

\section{Introduction}

Low energy effective actions for type II strings on a Calabi-Yau (CY) threefold are determined by $D=4 N=2$ supergravity actions coupled to vector- and hypermultiplets, or multiplets that can be dualized into these. The number of such multiplets depends on the Hodge numbers of the CY, and is respectively $h_{1,1}\left(h_{1,2}\right)$ and $h_{1,2}+1\left(h_{1,1}+1\right)$ for type IIA(B). The hypermultiplet moduli space is quaternion-Kähler and contains the dilaton. Therefore this space receives quantum corrections, both perturbatively and nonperturbatively. In this note, we focus on rigid $\left(h_{1,2}=0\right) \mathrm{CY}$ threefold compactifications of type IIA, or its mirror version in type IIB, understood in terms of a Landau-Ginzburg

†Talk given by S. Vandoren. To appear in the proceedings of the 36th International Symposium Ahrenshoop on the Theory of Elementary Particles, August 2003. 
orbifold [1]. Then there is only a single hypermultiplet, the universal hypermultiplet, whose tree level effective action is determined by the quaternion-Kähler target space [2, 3]

$$
\mathcal{M}_{H}=\frac{\mathrm{SU}(1,2)}{\mathrm{U}(2)} .
$$

This particular coset space is also Kähler, and there exist complex coordinates $S$ and $C$ in which the Kähler potential is given by

$$
K=-\ln (S+\bar{S}-2 C \bar{C})
$$

An alternative parametrization of the universal hypermultiplet in terms of four real scalars is

$$
e^{-1} \mathcal{L}_{\mathrm{UH}}=-\frac{1}{2} \partial^{\mu} \phi \partial_{\mu} \phi-\frac{1}{2} \mathrm{e}^{-\phi}\left(\partial^{\mu} \chi \partial_{\mu} \chi+\partial^{\mu} \varphi \partial_{\mu} \varphi\right)-\frac{1}{2} \mathrm{e}^{-2 \phi}\left(\partial_{\mu} \sigma+\chi \partial_{\mu} \varphi\right)^{2} .
$$

The relation with the complex coordinates above is given by

$$
\begin{aligned}
\mathrm{e}^{\phi}=\frac{1}{2}(S+\bar{S}-2 C \bar{C}) & \chi & =C+\bar{C}, \\
\sigma=\frac{\mathrm{i}}{2}\left(S-\bar{S}+C^{2}-\bar{C}^{2}\right) & \varphi & =-\mathrm{i}(C-\bar{C}) .
\end{aligned}
$$

Recently, the perturbative analysis of [4, 5] was revisited in [6], and it was shown that non-trivial perturbative quantum corrections only appear at one-loop. We will not discuss these corrections here, but only mention that they were found by studying deformations of (1.1) that preserve the Heisenberg subgroup of the classical group of $\mathrm{SU}(1,2)$ isometries

$$
S \rightarrow S+\mathrm{i} \alpha+2 \bar{\epsilon} C+|\epsilon|^{2}, \quad C \rightarrow C+\epsilon
$$

The parameters $\alpha$ and $\epsilon$ are real and complex respectively and, under the assumption that these transformations do not receive any quantum corrections, generate the symmetries that are preserved in string perturbation theory [4. In the basis of the real variables (1.4), the Heisenberg algebra of infinitesimal transformations is generated by

$$
\delta \phi=0, \quad \delta \chi=\epsilon+\bar{\epsilon}, \quad \delta \varphi=-\mathrm{i}(\epsilon-\bar{\epsilon}), \quad \delta \sigma=-\alpha-(\epsilon+\bar{\epsilon}) \varphi
$$

One can therefore choose a set of two commuting isometries, corresponding to the parameters $\alpha$ and $\epsilon=\mathrm{i} \beta$ (with $\beta$ real), to dualize the pseudoscalars $\varphi$ and $\sigma$ into two tensors, using the known Legendre transformation techniques. In such a double-tensor multiplet formulation, perturbative corrections are highly constrained due to the rather restrictive couplings of scalar-tensor systems with $N=2$ supersymmetry [7].

After dualization, the resulting tree level double-tensor multiplet Lagrangian reads [8, 17]

$$
e^{-1} \mathcal{L}_{\mathrm{DT}}=-\frac{1}{2} \partial^{\mu} \phi \partial_{\mu} \phi-\frac{1}{2} \mathrm{e}^{-\phi} \partial^{\mu} \chi \partial_{\mu} \chi+\frac{1}{2} M^{I J} H_{I}^{\mu} H_{\mu J}
$$


where the $H_{I}$ are a pair of three-form field strengths, $H_{I}^{\mu}=\frac{1}{2} \varepsilon^{\mu \nu \rho \sigma} \partial_{\nu} B_{\rho \sigma I}$, and

$$
M=\mathrm{e}^{\phi}\left(\begin{array}{cc}
1 & -\chi \\
-\chi & \mathrm{e}^{\phi}+\chi^{2}
\end{array}\right)
$$

The two scalars $\phi$ and $\chi$ parameterize the coset $\mathrm{SL}(2, \mathbb{R}) / \mathrm{O}(2)$. The presence of the tensors breaks the $\mathrm{SL}(2, \mathbb{R})$ symmetries to a two-dimensional subgroup generated by a certain rescaling of the fields and by the remaining generator of the Heisenberg algebra (1.6) with real parameter $\epsilon=\gamma / 2$. It acts as a shift on $\chi$ and transforms the tensors linearly into each other [8],

$$
\chi \rightarrow \chi+\gamma, \quad B_{1} \rightarrow B_{1}+\gamma B_{2},
$$

with $\phi$ and $B_{2}$ invariant. An invariant combination is then the tensor $\hat{H}_{1}=H_{1}-\chi H_{2}$, and we will call this the Heisenberg invariant. An off-shell superspace formulation of the Lagrangian (1.7) was given in [9], in terms of a single function satisfying a linear second order differential equation. In that paper, the superconformal calculus was used to write down the action. Upon gauge-fixing the conformal symmetries, it yields (1.7) or, equivalently, the action given in terms of the Calderbank-Pedersen variables [10].

In this note, we elaborate on [8], and investigate the non-perturbative effects that contribute to the low energy effective action. As explained in [11, these arise from Euclidean branes wrapped around supersymmetric cycles in the CY. In the case of IIA with $h_{1,2}=0$, the NS-fivebrane can wrap the entire CY, or the D2-brane can wrap a non-trivial three-cycle inside the CY. From the four-dimensional point of view, such configurations are localized in space and time and correspond to fivebrane and membrane instantons of the $N=2$ $D=4$ supergravity action.

In [8], following previous work of [12], the Bogomol'nyi equations were derived from (1.7). The solutions were shown to describe fivebrane and membrane-like instantons, and we review and extend this below. The natural description of these instantons was given in terms of the Euclidean continuation of the double-tensor multiplet action. This has the advantage over the Euclidean universal hypermultiplet that the Euclidean action is semipositive definite and hence it justifies the semi-classical approximation [8]. Perturbatively, the double-tensor multiplet guarantees $\mathrm{U}(1) \times \mathrm{U}(1)$ isometries in the dual hypermultiplet description. Non-perturbatively, however, the duality is expected to involve also the constant modes of the dual scalars $\varphi$ and $\sigma$ by means of theta-angle-like terms. Such terms break the $\mathrm{U}(1)$ isometries to a discrete subgroup, see e.g. 13, 14. 


\section{Fivebrane Instantons}

The first Bogomol'nyi equation that can be derived from the double-tensor multiplet action is given by $[8$

$$
\left(\begin{array}{c}
H_{\mu 1} \\
H_{\mu 2}
\end{array}\right)= \pm \partial_{\mu}\left(\begin{array}{c}
\mathrm{e}^{-\phi} \chi \\
\mathrm{e}^{-\phi}
\end{array}\right)
$$

where the plus and minus signs refer to instantons and anti-instantons, respectively. The closure of the three-form field strengths then implies Laplace-like equations for the scalars. On a flat spacetime $\mathcal{M}$ with points $\left\{x_{i}\right\}$ excised from $\mathbb{R}^{4}$, we find the multi-centered solutions in terms of two harmonic functions,

$$
\mathrm{e}^{-\phi}=\mathrm{e}^{-\phi_{\infty}}+\sum_{i} \frac{\left|Q_{2 i}\right|}{4 \pi^{2}\left(x-x_{i}\right)^{2}}, \quad \mathrm{e}^{-\phi} \chi=\mathrm{e}^{-\phi_{\infty}} \chi_{\infty}+\sum_{i} \frac{Q_{1 i}}{4 \pi^{2}\left(x-x_{i}\right)^{2}}
$$

where $Q_{1 i}, Q_{2 i}, \chi_{\infty}$, and $\phi_{\infty}$ are independent integration constants; the latter two determine the asymptotic values of the fields at infinity. We wrote the absolute value of $Q_{2 i}$ to make $\mathrm{e}^{-\phi}$ positive everywhere in space, and we identify the string coupling constant via $g_{s}=\mathrm{e}^{-\phi_{\infty} / 2}$. Furthermore, two charges are defined by integrating the tensor field strengths $H_{\mu \nu \rho I}=-\varepsilon_{\mu \nu \rho \sigma} H_{I}^{\sigma}$ over 3-spheres at infinity,

$$
Q_{I}=\int_{S_{\infty}^{3}} H_{I}, \quad I=1,2
$$

They are related to the constants appearing in the scalar fields through the field equation (2.1). Using $* *=-1$ on a three-form in four Euclidean dimensions, we find

$$
Q_{2}=\mp \sum_{i}\left|Q_{2 i}\right|, \quad Q_{1}=\mp \sum_{i} Q_{1 i}
$$

This implies that for instantons, $Q_{2}$ should be taken negative, whereas for anti-instantons, $Q_{2}$ must be positive. Note that there is no restriction on the sign of the $Q_{1 i}$.

The (anti-) instanton action for the fivebrane can be computed using the formulas in [8]. It is finite only if $\chi$ remains finite near the excised points $x_{i}$. In the limit, we find

$$
\chi_{i} \equiv \lim _{x \rightarrow x_{i}} \chi(x)=\frac{Q_{1 i}}{\left|Q_{2 i}\right|},
$$

which is finite whenever $Q_{2 i} \neq 0$ for nonvanishing $Q_{1 i}$. This implies that the integrated Heisenberg invariants vanish, $\hat{Q}_{1 i} \equiv Q_{1 i}-\chi_{i}\left|Q_{2 i}\right|=0$. Plugging the solution into the action, we find

$$
S_{\text {inst }}=\frac{\left|Q_{2}\right|}{g_{s}^{2}}+\frac{1}{2} \sum_{i}\left|Q_{2 i}\right|\left(\chi_{\infty}-\chi_{i}\right)^{2}
$$


The quadratic dependence on the string coupling constant is precisely what corresponds to a wrapped NS-fivebrane [1].

For a single-centered instanton around $x_{0}$, this reduces to

$$
S_{\mathrm{inst}}=\frac{\left|Q_{2}\right|}{g_{s}^{2}}\left(1+\frac{1}{2} g_{s}^{2}(\Delta \chi)^{2}\right),
$$

where $\Delta \chi=\chi_{\infty}-\chi_{0}$ is the difference between the values of the $\mathrm{R}$ - $\mathrm{R}$ scalar at the boundaries $S_{\infty}^{3} \cup S_{0}^{3}$ of $\mathbb{R}^{4}-\left\{x_{0}\right\}$. It would be interesting to have a better string theoretic interpretation of this term.

An important issue is whether our solutions (2.2) preserve half of the supersymmetry. This question is analyzed in detail in [15]. It turns out that the spherically symmetric solutions are BPS in the sense of preserving four supercharges. Interestingly, the general multi-centered solution satisfying the Bogomol'nyi bound (2.1) is not, and imposing the BPS condition leads to the further restriction that all the $\chi_{i}$ must be equal. This implies that the solution is characterized in terms of a single harmonic function, given by $\mathrm{e}^{-\phi}$. When all $\chi_{i}$ are equal, the value of the action is lowered and coincides with the spherically symmetric case (2.7). A possible interpretation is that the general multi-centered solution is metastable and decays into the state where all $\chi_{i}$ are equal. In such a state, the points $x_{i}$ can be brought together to a spherically symmetric configuration without changing the action. We call the general solution (2.2) near-extremal when the values of $\chi_{\infty}-\chi_{i}$ are all close to the lowest value $\Delta \chi$.

\section{Membrane Instantons}

The second Bogomol'nyi equation that can be derived from (1.7) contains an arbitrary constant $\chi_{0}[8$,

$$
\left(\begin{array}{c}
H_{\mu 1} \\
H_{\mu 2}
\end{array}\right)= \pm \frac{1}{\left|\tau^{\prime}\right|}\left(\begin{array}{c}
\chi\left(\chi-\chi_{0}\right) \partial_{\mu} \mathrm{e}^{-\phi}+\mathrm{e}^{-\phi}\left(\chi+\chi_{0}\right) \partial_{\mu} \chi+2 \mathrm{e}^{\phi} \partial_{\mu} \mathrm{e}^{-\phi} \\
\left(\chi-\chi_{0}\right) \partial_{\mu} \mathrm{e}^{-\phi}+2 \mathrm{e}^{-\phi} \partial_{\mu} \chi
\end{array}\right)
$$

where $\tau^{\prime}=\left(\chi-\chi_{0}\right)+2 \mathrm{ie}^{\phi / 2}$. Below, for the single-centered solution, we will identify $\chi_{0}$ with the value of $\chi$ at the excised point. To solve these equations, we first consider the combination

$$
H_{\mu 1}-\chi_{0} H_{\mu 2}= \pm \partial_{\mu}\left(\mathrm{e}^{-\phi}\left|\tau^{\prime}\right|\right)
$$

The Bianchi identities then imply that $h=\mathrm{e}^{-\phi}\left|\tau^{\prime}\right|$ is harmonic and positive everywhere. With $\chi$ expressed in terms of $h$ and $\phi$,

$$
\chi-\chi_{0}=\mathrm{e}^{\phi} \sqrt{h^{2}-4 \mathrm{e}^{-\phi}},
$$


the condition for $H_{\mu 2}$ turns into

$$
H_{\mu 2}= \pm \frac{1}{\sqrt{h^{2}-4 \mathrm{e}^{-\phi}}}\left(2 \mathrm{e}^{-\phi} \partial_{\mu} h-h \partial_{\mu} \mathrm{e}^{-\phi}\right)
$$

Notice that we have taken the positive branch in (3.3), the negative branch just changes the sign in (3.4). The Bianchi identity and the harmonic property of $h$ now imply that

$$
\left(h^{2}-4 \mathrm{e}^{-\phi}\right) \partial_{\mu} \partial^{\mu} \mathrm{e}^{-\phi}+2 \partial_{\mu} \mathrm{e}^{-\phi} \partial^{\mu} \mathrm{e}^{-\phi}-2 h \partial_{\mu} h \partial^{\mu} e^{-\phi}+2 \mathrm{e}^{-\phi} \partial_{\mu} h \partial^{\mu} h=0 .
$$

It is unclear how to solve this equation in general, but some multi-centered solutions were constructed in 16. To continue, we assume that the dilaton depends on the coordinates only through $h$. This assumption is justified if we restrict ourselves to spherically symmetric configurations,

$$
h=\mathrm{e}^{-\phi_{\infty}}\left|\tau_{\infty}^{\prime}\right|+\frac{\left|\hat{Q}_{1}\right|}{4 \pi^{2}\left(x-x_{0}\right)^{2}},
$$

where $\hat{Q}_{1}=Q_{1}-\chi_{0} Q_{2}$, which must be taken negative for instantons (upper sign in (3.11)). We now proceed to find the most general spherically symmetric solution of (3.5), extending the special cases of [8]. By differentiating (3.5]) once more, one can solve for the dilaton in terms of three integration constants,

$$
\mathrm{e}^{-\phi}=a h^{2}+b h+c
$$

Combining this with equations (3.4) and (3.5), we find that $c=-\beta^{2}$, where $\beta \equiv \pm Q_{2} /\left|\hat{Q}_{1}\right|$, and $b=-\beta \sqrt{1-\alpha}$, where $a=\alpha / 4$. Positivity of the dilaton and reality of $H_{2}$ requires furthermore $0 \leq \alpha \leq 1$, but the case $\alpha=0$ must be treated separately and in fact precisely coincides with the fivebrane instanton solution. Plugging (3.7) into (3.3), and evaluating it at infinity, one finds that $\alpha$ is fixed in terms of the charges and the asymptotic values of the fields,

$$
\alpha=1-\frac{\left(\Delta \chi-2 \beta \mathrm{e}^{\phi_{\infty}}\right)^{2}}{\left|\tau_{\infty}^{\prime}\right|^{2}}
$$

where $\Delta \chi=\chi_{\infty}-\chi_{0}$. The solution for $\chi$ can be read off from (3.3) and one can check that $\chi_{0}$ is indeed the value at the origin. An important difference with the fivebrane is that $\chi$ needs a source at the excised point. Positivity of $\alpha$ forces, for fixed $g_{s}$ and $\Delta \chi$ (positive), $\beta$ to be in the interval

$$
\frac{\Delta \chi-\left|\tau_{\infty}^{\prime}\right|}{2 \mathrm{e}^{\phi_{\infty}}} \leq \beta \leq \frac{\Delta \chi+\left|\tau_{\infty}^{\prime}\right|}{2 \mathrm{e}^{\phi_{\infty}}}
$$

The complete solution is given in terms of one harmonic function, and all the constants $a$, $b, c$ are fixed in terms of the charges and the asymptotic values of the scalars. We have checked, using the supersymmetry transformation rules given in [7, 15], that the solution 
preserves half of the supersymmetry (except in the limit $\alpha \rightarrow 0$ ), and hence is truly BPS. The instanton action can be computed using the formulas of [8], and equals

$$
S_{\mathrm{inst}}=\left|\tau_{\infty}^{\prime}\right|\left(\left|\hat{Q}_{1}\right|+\frac{1}{2} \Delta \chi Q_{2}\right)
$$

Positivity of the action is guaranteed by the bounds (3.9). For $Q_{2}=0$, which trivially satisfies (3.9), we find instanton actions which are, for $\Delta \chi=0$, inversely proportional to the string coupling constant, $S_{\text {inst }}=2\left|Q_{1}\right| / g_{s}$. This is the correct $g_{s}$-dependence of a membrane instanton with charge $Q_{1}$ [1]. Adding fivebrane charge $Q_{2}$ raises the action until, for fixed $g_{s}$ and $\Delta \chi$, one reaches the $\alpha \rightarrow 0$ bound (3.9). At that point, the solution is no longer stable, and should be replaced by the fivebrane instanton.

\section{Acknowledgement}

S.V. would like to thank the organizers of the Simons Workshop in Mathematics and Physics, Stony Brook 2003, and those of the Ahrenshoop Symposium, Wernsdorf 2003, for their hospitality and stimulating meetings. We have also benefited from discussions with many of the participants.

\section{References}

[1] P.S. Aspinwall and B.R. Greene, On the geometric interpretation of $N=2$ superconformal theories. Nucl. Phys. B437 (1995) 205, hep-th/9409110.

[2] S. Cecotti, S. Ferrara and L. Girardello, Geometry of type II superstrings and the moduli of superconformal field theories. Int. J. Mod. Phys. A4 (1989) 2475.

[3] S. Ferrara and S. Sabharwal, Dimensional reduction of type II superstrings. Class. Quant. Grav. 6 (1989) L77; Quaternionic manifolds for type II superstring vacua of Calabi-Yau spaces. Nucl. Phys. B332 (1990) 317.

[4] A. Strominger, Loop corrections to the universal hypermultiplet. Phys. Lett. B421 (1998) 139, hep-th/9706195.

[5] H. Günther, C. Herrmann and J. Louis, Quantum corrections in the hypermultiplet moduli space, Fortsch. Phys. 48 (2000) 119, hep-th/9901137.

[6] I. Antoniadis, R. Minasian, S. Theisen and P. Vanhove, String loop corrections to the universal hypermultiplet. hep-th/0307268.

[7] U. Theis and S. Vandoren, $N=2$ supersymmetric scalar-tensor couplings. JHEP 0304 (2003) 042, hep-th/0303048. 
[8] U. Theis and S. Vandoren, Instantons in the double-tensor multiplet. JHEP 0209 (2002) 059, hep-th/0208145.

[9] B. de Wit, M. Roček and S. Vandoren, Hypermultiplets, hyperkähler cones, and quaternion-Kähler geometry. JHEP 0102 (2001) 039, hep-th/0101161.

[10] D. Calderbank and H. Pedersen, Selfdual Einstein metrics with torus symmetry. J. Diff. Geom. 60 (2002) 485, math.DG/0105263.

[11] K. Becker, M. Becker and A. Strominger, Fivebranes, membranes and non-perturbative string theory. Nucl. Phys. B456 (1995) 130, hep-th/9507158.

[12] M. Gutperle and M. Spalinski, Supergravity instantons and the universal hypermultiplet. JHEP 0006 (2000) 037, hep-th/0005068; Supergravity instantons for N=2 hypermultiplets. Nucl. Phys. B598 (2001) 509, hep-th/0010192.

[13] H. Ooguri and C. Vafa, Summing up D-instantons. Phys. Rev. Lett. 77 (1996) 3296, hep-th/9608079.

[14] S. Ketov, Summing up D-instantons in N=2 supergravity. Nucl. Phys. B649 (2003) 365, hep-th/0209003.

[15] M. Davidse, U. Theis and S. Vandoren, Fivebrane instantons in $D=4 \mathrm{~N}=2$ supergravity. In preparation.

[16] M. de Vroome, Instantons and branes in low energy superstring theory. Masters thesis, Utrecht, March 2003. 